\title{
DIAMAGNETIC PLASMOIDS AS PART OF DIAMAGNETIC STRUCTURES OF THE SLOW SOLAR WIND AND THEIR IMPACT ON EARTH'S MAGNETOSPHERE
}

\author{
V.A. Parkhomov \\ Baikal State University, \\ Irkutsk, Russia, pekines_41@mail.ru \\ V.G. Eselevich \\ Institute of Solar-Terrestrial Physics SB RAS, \\ Irkutsk,Russia,esel@iszf.irk.ru \\ M.V. Eselevich \\ Institute of Solar-Terrestrial Physics SB RAS, \\ Irkutsk,Russia,mesel@iszf.irk.ru
}

\author{
A.V. Dmitriev \\ Skobeltsyn Institute of Nuclear Physics, \\ Moscow State University, \\ Moscow,Russia,dalexav@mail.ru \\ T.I. Vedernikova \\ Baikal State University, \\ Irkutsk,Russia,vedernikovati@bgu.ru
}

\begin{abstract}
We have shown that diamagnetic structures (DSs), which form the basis of the slow quasistationary solar wind (SW), are observed in Earth's orbit as a sequence of DSs of various scales. The analysis of this phenomenon indicates that diamagnetic plasmoids in SW, whose concept was introduced by Karlsson in 2015, are identical to small-scale DSs. We have found that the impact of a sequence of DSs in the slow SW on Earth's magnetosphere causes an increase in geomagnetic activity. Isolated DSs generate short-term magnetic disturbances whose duration is approximately
\end{abstract}

equal to the DS duration. Hence, a sequence of DSs can cause sawtooth substorms. We emphasize that the interaction of DS in the slow SW under northward interplanetary magnetic field can be associated with penetration of DS high-density plasma into the magnetosphere.

Keywords: diamagnetic structures of solar wind, diamagnetic plasmoids, streamer chains, sawtooth substorms.

\section{INTRODUCTION}

This article deals with the peculiarities of interaction between Earth's magnetosphere and the quasi-stationary solar wind $(\mathrm{SW})$ of a certain type - slow SW. In this regard, we present a brief overview on types of SW streams and on sources of these streams on the Sun.

The quasi-stationary SW represents streams the lifetime of sources of which on the Sun is longer or much longer than the day. The lifetime of sources of the sporadic SW, associated with coronal mass ejections (CMEs), is shorter than the day. In the absence of the sporadic SW, the quasi-stationary SW can be of two types: fast and slow. Sources of the slow quasistationary SW on the Sun are streamer belt [Svalgaard, 1974] and chains [Eselevich et al., 2007; Eselevich, 1999], or pseudostreamers [Wang, 2007]. In Earth's orbit, the slow SW has a relatively low velocity $V \approx$ $250-450 \mathrm{~km} / \mathrm{s}$ as compared to the fast quasi-stationary SW flowing from coronal holes whose maximum velocity $V \sim 450-800 \mathrm{~km} / \mathrm{s}$ [Borrini et al., 1981; Eselevich, Fainshtein, 1991, 1992].

One of the two above sources of the slow SW on the solar surface - streamer belt - has the following feature. It separates regions with the oppositely directed radial component of the global magnetic field [Svalgaard et al., 1974]. This means that at the base of the streamer belt are magnetic arches forming the base of the streamer helmet through tops of which the neutral line (NL) of the radial component of the global solar magnetic field runs [Eselevich et al., 1999]. The structure of the coronal streamer belt above the helmet represents a sequence of pairs of higher brightness rays (plasma density) or two closely spaced series of rays. The angular size of all the rays is equal $\left(d \sim 2^{\circ}-3^{\circ}\right)$, the distance between the rays along the streamer belt is $5^{\circ}-$ $10^{\circ}$. (Hereinafter, the angular size is given in the heliocentric coordinate system). NL passes between rays of each pair along the belt [Eselevich, Eselevich, 2006]. The streamer belt encircles the Sun as a wave-like surface (skirt) [Korzhov, 1977]. The streamer belt extension in the heliosphere (slow SW) is called heliospheric plasma sheet (HPS) [Winterhalter et al., 1994]. The streamer belt NL inside HPS in Earth's orbit appears as a sector boundary of the interplanetary magnetic field (IMF) separating extended SW regions (of the order of tens of degrees in the heliocentric coordinate system or, respectively, of several days) with opposite IMF signs [Korzhov, 1977]. The positive antisunward direction corresponds to the azimuth angle $\Phi<180^{\circ}$; the negative one, to $\Phi>180^{\circ}$. There is therefore an odd number of reversals of IMF sign (if it is greater than unity) in the structure of the sector boundary.

The angular size and relative changes of density observed near the Sun in ray structures of the streamer belt persist all the way from the Sun to Earth's orbit [Eselevich et al., 2006]. Here, these HPS areas have the following parameters and features [Borrini et al., 1981; Eselevich, Eselevich, 2006]: 
- relatively small SW velocity $V \sim 250-450 \mathrm{~km} / \mathrm{s}$, which is nearly constant through the layer;

- lower proton temperature $T_{\mathrm{p}}<10^{5} \mathrm{~K}$;

- higher plasma density with maximum values $N$ $\max >8 \mathrm{~cm}^{-3}$;

- anticorrelation of density change $N(t)$ and magnetic field modulus $B(t)$ on time scales of, at least, the order of hours or smaller;

- the presence of one or several (odd number) IMF sign reversals, which are a manifestation of the sector boundary or its structure.

The presence of all these features is required for unambiguous identification of the slow SW flowing in HPS in Earth's orbit.

Streamer chains (pseudostreamers) in the white light corona, similar to the streamer belt, have the form of a sequence of higher brightness rays (plasma density) with the same angular size. In them, the slow SW has approximately the same properties as in the streamer belt. The streamer chains differ from the belt in the fact that they separate coronal regions with open magnetic field lines having the same polarity. The magnetic field structures at the base of the chains on the solar surface, therefore, have the form of double arches [Eselevich et al., 1999].

In Earth's orbit, the streamer chains are recorded, as is the case with the streamer belt, in the form of areas with increased plasma density but containing an even number of IMF sign reversals. These areas are referred to as subsector boundaries [Ivanov et al., 2002].

The analysis carried out in [Eselevich, Eselevich, 2005] has led to the conclusion that the antisunward extensions of higher brightness rays of the streamer belt (HPS) and chains recorded in Earth's orbit are diamagnetic tubes or diamagnetic structures (DS). Eselevich, Eselevich [2005] have also shown that DSs have a fine structure (fractality) on several spatial scales from $\sim 1.5^{\circ}-3.0^{\circ}$ to the minimum scale $\sim 0.03^{\circ}-0.06^{\circ}$. The angular size of embedded magnetic tubes is seen to vary by almost two orders of magnitude.

The first attempts to examine collisions of slow-SW DSs with Earth's magnetosphere have shown that they can cause so-called sawtooth substorms [Parkhomov et al., 2011; Parkhomov et al., 2018].

All the results reflect the foundations of the modern understanding of the nature of the structure of the slow SW all the way from the Sun to Earth's orbit.

At the same time, in a number of papers published abroad over the last few years (2012-2017) without references to the above results, conclusions have been drawn about a new type of plasma density irregularities in the quasi-stationary SW. For example, Karlsson et al. [2012, 2015] assert that in the quasi-stationary SW there are density irregularities called SW plasmoids. The authors identify them as a local increase in plasma density (>50\%) $N$ with simultaneously decreasing magnetic field modulus $B$ and a constant velocity $V_{\mathrm{SW}}$ on a scale from 0.1 to $10 R_{\mathrm{E}}\left(R_{\mathrm{E}}\right.$ is the Earth radius). The anticorrelation of $N$ and $B$ variations served as the basis for the term SW diamagnetic plasmoid. The constant velocity inside and outside the plasmoid implies that it is carried away by the slow SW. The duration of the diamagnetic plasmoid is $\sim 5-10$ min [Karlsson et al., 2015]. In addition to SW plasmoids, the authors have detected diamagnetic plasmoids behind the bow near-Earth shock wave in the magnetosheath (magnetosheath plasmoids), which have approximately the same size from 0.1 to $10 R_{\mathrm{E}}$. The authors have not, however, found a direct connection between plasmoids in SW and magnetosheath. The question also remains unanswered about the impact of the magnetosheath plasmoid on Earth's magnetosphere.

The purpose of this paper is, first, to conduct an analysis in order to clarify the physical meaning of the term SW diamagnetic plasmoid and its relationship with DS of the slow SW; second, to study the role of SW diamagnetic plasmoids in forming magnetosheath plasmoids; and third, to analyze results of their impact on Earth's magnetosphere.

\section{DATA AND METHODS}

The solar source of the slow SW in the March 11, 2003 event has been identified using the synoptic map of Carrington rotation CR2000 for the solar magnetic field, calculated in the potential approximation [http://wso.stan-ford.edu/]. In the July 24, 1998 event, the position of the solar source of the slow SW was determined from the calculations made by Rudenko [http://bdm.iszf.irk.ru] in the potential approximation for CR1938. Areas of the slow SW in Earth's orbit were analyzed from time dependences of IMF and SW plasma parameters derived from OMNI data [http://cdaweb.gsfc.nasa.gov/cgi-bin/eval2.cgi]. To calculate the time of arrival of the area of the slow SW from the solar source at Earth's orbit, we have used the method described in [Eselevich et al., 2007]. When analyzing the response of the magnetosphere to the impact of SW streams, we have employed the following data: a) magnetograms from the Barrow Observatory (MLT= UT-12) and the dynamic spectrum of geomagnetic pulsations derived from low-latitude Memambetsu Observatory data $(\mathrm{MLT}=\mathrm{UT}+9)$; b) the dynamic spectrum of geomagnetic pulsations obtained from Kakioka Observatory data $(\mathrm{KAK})$; c) images of the auroral oval taken by the UVI camera with the LBHL filter on board the Polar satellite on August 01, 1998.

\section{RESULTS}

Identification of an area of the slow $S W$ related to the streamer belt and its solar source in Earth's orbit on March 11, 2003. In Figure 1, the vertical rectangle marks an area in Earth's orbit that includes the March 11, $2003 \mathrm{SW}$ diamagnetic plasmoid. This area has the following features.

1. Increased plasma density $N>10 \pm 2 \mathrm{~cm}^{-3}$.

2. Low SW velocity $V \sim 390 \mathrm{~km} / \mathrm{s}$.

3. The presence of the IMF sector boundary within this area at which the azimuth angle $\Phi$ changes by $\sim 180^{\circ}$ (from $\sim 320^{\circ}$ to $\sim 140^{\circ}$ ), i.e. the IMF sign changes from "-" (sunward) to "+" (antisunward).

These features characterize the area of interest as being part of the streamer belt in Earth's orbit (HPS). On the solar surface, the source of this HPS area should be 


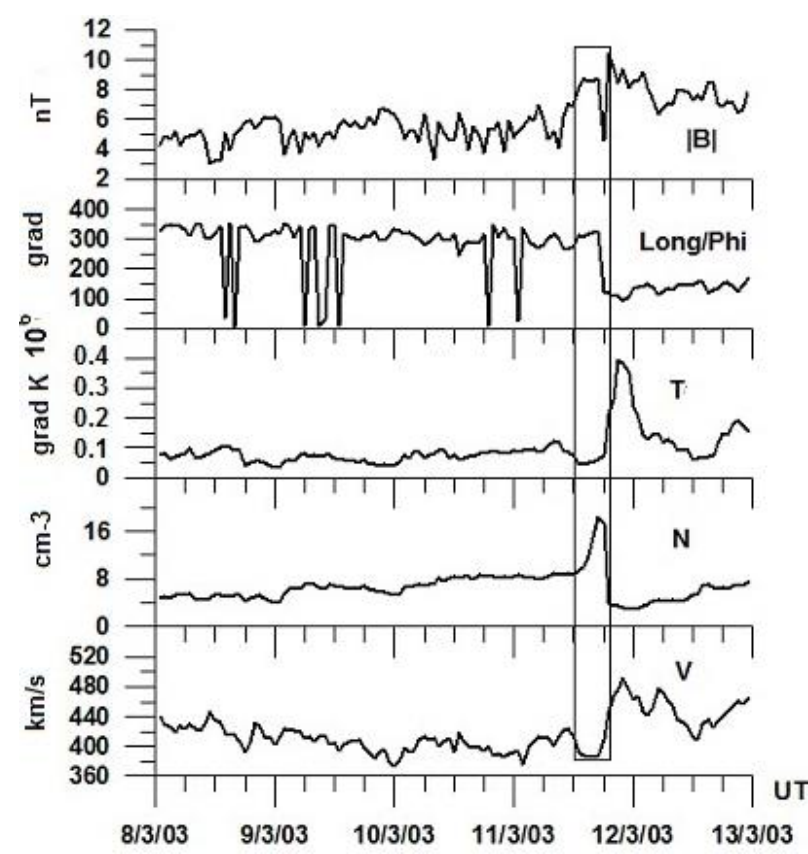

Figure 1. Parameters of IMF and SW plasma. From top to bottom: IMF modulus, IMF azimuth angle $\Phi, \mathrm{SW}$ plasma temperature, SW plasma density, SW velocity. The rectangle marks a streamer belt area in Earth's orbit or HPS. OMNI data [http://cdaweb.gsfc.nasa.gov/cgi-bin/eval2.cgi]

in the vicinity of the point of intersection of the streamer belt with the ecliptic when it crosses the central meridian due to the solar rotation. Indeed, on the synoptic map (Figure 2) to the streamer belt corresponds NL of the global magnetic field, which separates the positive (solid curves) and negative (dotted line) field polarities. We can see that the central meridian passes in the vicinity of the point $\mathrm{O}$ of intersection of NL with the ecliptic ( horizontal line) on March 07, 2003 at $t_{0} \approx 12: 00$ UT (vertical line). This area is a source of slow SW in the streamer belt. We can demonstrate this by estimating the time $t_{\text {Earth }}$ of the arrival of the belt area of interest at Earth's orbit from the formula [Eselevich et al., 2007]

$$
t_{\text {Earth }} \approx t_{0}+4.6 \cdot 10^{4} / \mathrm{V}, \mathrm{hr},
$$

where $t_{0}$ is the time (hr) of the passage of the point $\mathrm{O}$ through the central meridian (Figure 2) over a distance $(\mathrm{km}), V$ is the velocity of the slow $\mathrm{SW}$ at $1 A U(\mathrm{~km} / \mathrm{s})$.

Referring to Figure 1, in Earth's orbit the velocity of the slow SW in the streamer belt $V \sim 390 \mathrm{~km} / \mathrm{s}$. Then the time $\Delta t_{\text {Earth }}$ the solar wind passes the Earth-Sun distance in accordance with the above formula is estimated at $\sim 4$ days $22 \mathrm{hrs}$, which corresponds to the time of its arrival at Earth's orbit on March 12, 2003 ( $10 \mathrm{hrs})$. Within the accuracy of 1 day (or relative accuracy of $\approx 20 \%$ ) this is consistent with the time of arrival of the area at Earth's orbit with the IMF azimuth angle $\Phi$ changing sing, i.e. sector boundary.

It passes within the HPS area whose maximum plasma density $N \sim 18 \mathrm{~cm}^{-3}$. The change of the magnetic field sign near the Sun from "-" to «+» (Long/ Phi panel) corresponds to the change of the angle $\Phi$ sign. The HPS region is marked with a rectangle.

Relationship between the March 11, 2003 SW diamagnetic plasmoid and DS of the slow SW. The choice of the date March 11, 2003 is explained by the fact that on that day, according to [Karlsson et al., 2015], an SW diamagnetic plasmoid was identified in the slow SW. The basis for the introduction of this term was the anticorrelation the authors found in the behavior of SW plasma density (sharp increase) and IMF strength (sharp decrease), as well as the reference to the similar results obtained in the experimental work [Bostick, 1956].

To find the relationship between the SW diamagnetic plasmoid and the slow SW, we examine the structure of the HPS region for March 11, 2003, shown in Figure 3.

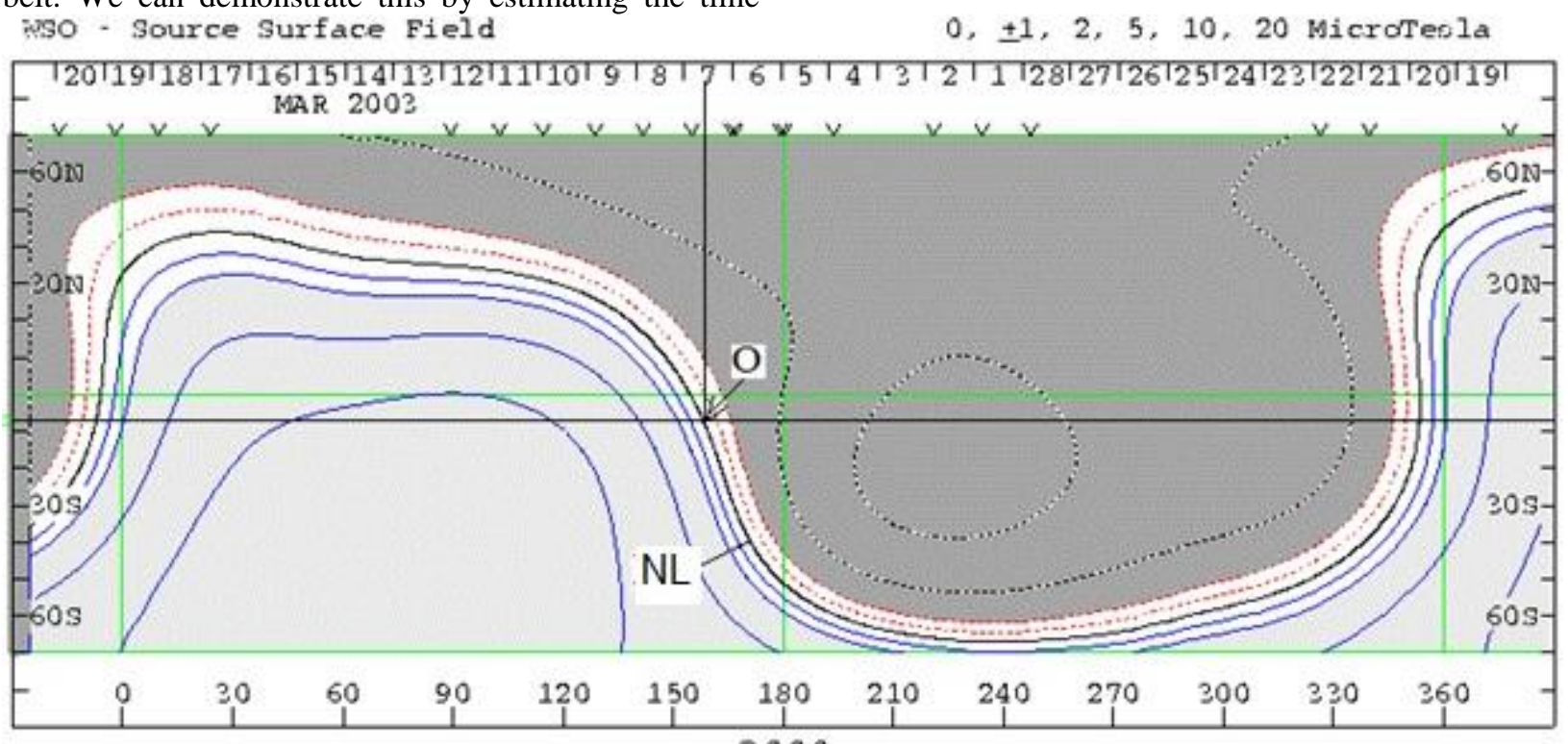

2000

Figure 2. Synoptic map of Carrington rotation 2000 for the solar magnetic field calculated in the potential approximation [http://wso.stanford.edu/]: solid curves indicate positive polarity; the dotted curve is negative polarity, NL is the neutral line of the global solar magnetic field 


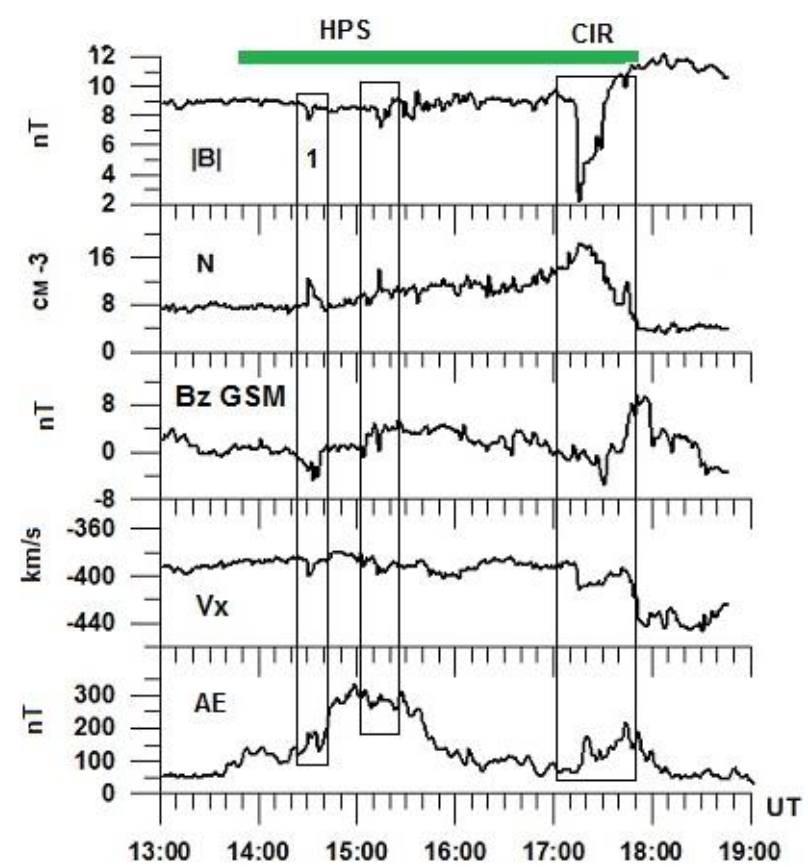

Figure 3. Parameters of IMF and SW plasma for March 11,2003 . From top to bottom: $B$ is the IMF modulus, $N$ is the SW plasma density, $B_{\mathrm{z}}$ is the IMF vertical component, $V_{x}$ is the $\mathrm{SW}$ velocity, $A E$ is the auroral magnetic activity index derived from OMNI data [http://cdaweb.gsfc.nasa.gov/cgibin/ eval2.cgi]. An area of SW diamagnetic plasmoid [Karlsson et al., 2015] is marked with rectangle 1

For this region on an increased time scale in addition to the IMF modulus $B$ and its $B$ z component in GSM coordinates, we present the SW velocity $V$, plasma density $N$, auroral magnetic activity index $A E$. Rectangle 1 indicates the March 11, 2003 SW diamagnetic plasmoid (14:31-14:35 UT), studied in [Karlsson et al., 2012]. The HPS region is shown by the horizontal line labeled HPS.
As seen from the comparison of $B$ and $N$ profiles, HPS is a sequence of DSs (marked with vertical rectangles $|\mathbf{B}|$ ) of different time scales. Some of them are poorly seen because of small time scales and insufficient temporal resolution. Maximum density values in these structures $\sim 18 \mathrm{~cm}^{-3}$ are achieved in the region of interaction between slow and fast SW whose right boundary is determined from increasing SW velocity at the end of HPS at $t>17: 45$ UT. This region is also called corotating interaction region (CIR) (marked with a rectangle). It represents DS within which DSs are recorded on several decreasing spatial scales. The HPS-mean anticorrelation coefficient between $N$ and $B$ is 0.746. According to [Eselevich, Eselevich, 2005], the embedded DSs of this type with decreasing transverse size characterize the presence of fractal structure in HPS.

Figure 4 shows variations of SW and IMF parameters derived from OMNI data and SW plasma and magnetic field parameters obtained from Cluster $\mathrm{C} 1$ observations [Karlsson et al., 2015]. One-to-one antiphase correspondence can be seen in the $N$ and $B$ variations. The differences in fine details are due to different temporal resolution of parameter measurements. In OMNI data, the temporal resolution is $1 \mathrm{~min}$, and measurement sampling frequency in Cluster satellites is $4.4 \mathrm{~s}$. Noteworthy is the delay of variations derived from Cluster $\mathrm{C} 1$ measurements relative to the beginning of variations in SW by $85 \mathrm{~s}$ according to OMNI data. Accuracy of data transfer from the Wind orbit to Earth's orbit is \pm 60 according to [King, Papitashvili, 2006]. In view of this comment, we can conclude that the structures shown by rectangles in the right and left panels are identical. We can therefore conclude that the plasmoid and DS recorded in SW represent the same structure called differently. Below we use our designation DS.

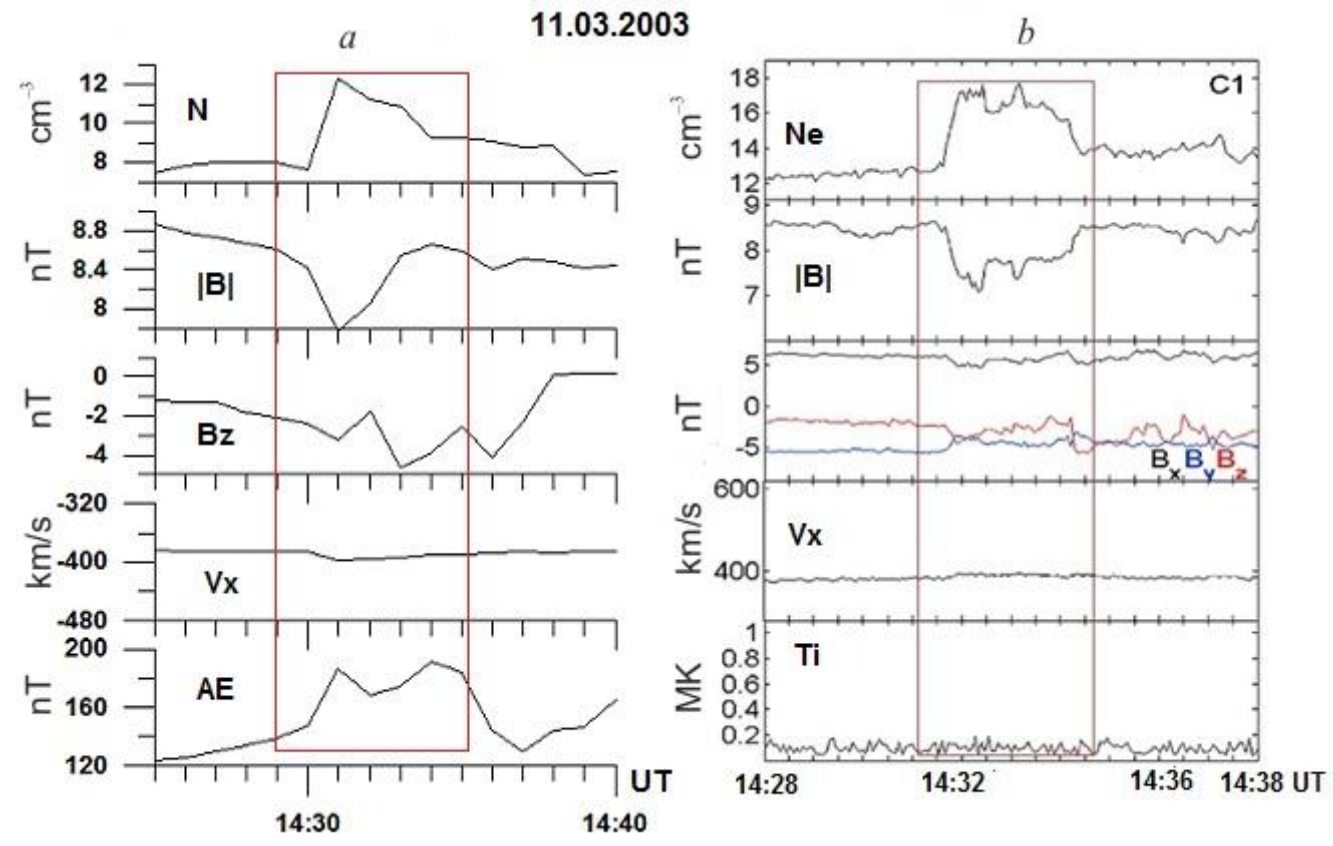

Figure 4. SW and IMF parameters on March 11, 2003 derived from OMNI data (a) and Cluster C1 measurements [Karlsson et al., 2015] (b). Rectangles mark DS according to [Eselevich, Eselevich, 2005] (a) and plasmoid according to [Karlsson et al., 2015] (b) 
A similar comparative analysis carried out for two more SW diamagnetic plasmoids in [Karlsson et al., 2015] for April 04, 2004 (12:24-12:30 UT) and April 05, 2006 (04:37-04:47 UT) also leads to a similar conclusion. Thus, we can confidently assert that the diamagnetic plasmoids observed in the slow SW, which have been discussed in [Karlsson et al., 2015], are internal elements of fractal DSs of the slow SW.

The question about the presence of DS in the fast SW has not been examined yet. Nevertheless, the SW diamagnetic plasmoid for January 24, 2004 (06:0006:06 UT) in [Karlsson et al., 2015] was detected in the fast SW. This means that, perhaps only some of the 62 SW diamagnetic plasmoids analyzed in [Karlsson et al., 2015] are associated with the slow SW, and therefore these plasmoids are elements of fractal DSs constituting the slow SW. Recently, Karlsson has kindly provided us with a list of events studied in [Karlsson et al., 2015]. Table 1 includes 57 events from this list, associated with the slow SW, with all parameters necessary for the analysis reliably recorded. Notes to Table: Column $1-$ number of event, Column 2 - date, Column 3 - time, Column 4 - SW velocity, Column 5 - SW type (slow SW), Column 6 - solar source of SW, Column 7 - mean $A E$ within 40 min before the moment of plasmoid detection / mean $A E$ within 5 min after the moment of detection, Column 8 - mean $B_{\mathrm{z}}$ (in the GSM coordinate system) within $40 \mathrm{~min}$ before the detection. The bottom row shows mean values for the entire Table.

From the analysis of Table we can make a tentative conclusion that during the interaction of the plamoids with the magnetosphere there is a jump of auroral magnetic activity by $\sim 93 \mathrm{nT}$, which corresponds to the weak substorm with a mean vertical component of $-0.73 \mathrm{nT}$. Note that 18 cases of plasmoids (32\%) have been recorded against the background of the northward IMF component coming before the event. The highest jumps of $A E$ at the time of plasmoid detection (to $250 \mathrm{nT}$ ) were, however, observed against the previous southward IMF component. This allows us to interpret the moment of interaction of the plasmoid with the magnetosphere as a trigger of the start of auroral activity increase.

\section{ANALYSIS OF INTERACTION OF THE MARCH 11, 2003 HPS AREA WITH THE MAGNETO- SPHERE}

The collision of a sequence of similar small DSs, which follow the DSs described above and form the base of HPS, with Earth's magnetosphere causes planetary and auroral geomagnetic activities to increase: $K_{\mathrm{p}}$ increases from 2+ to 4+, $A E$ smoothly increases from 50 to $350 \mathrm{nT}$ (Figure 3). Figure 4, $a$ shows an increase in $A E$ from 140 to $190 \mathrm{nT}$, which may be interpreted as a magnetospheric response to the interaction with DS. The auroral observatory BRW near midnight at 14:28 UT (02:28 MLT) recorded the beginning of a negative bay (Figure 5, a), and the low-latitude observatory of the night sector (MLT 23:28) observed the beginning of Pi2 (Figure 5, $b$ ).
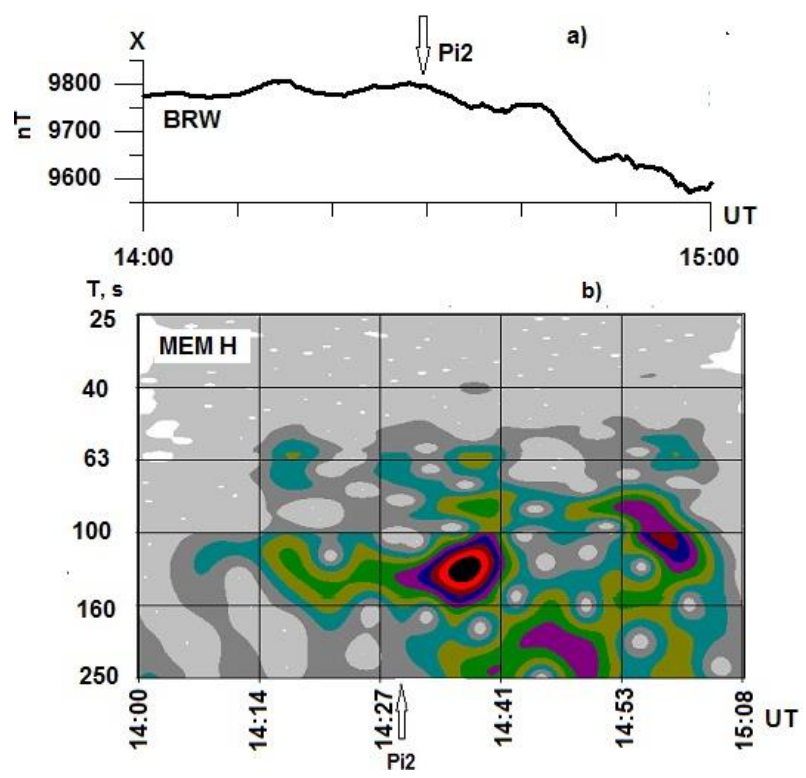

Figure 5. Magnetogram from the Barrow Observatory $(\mathrm{MLT}=\mathrm{UT}-12)(a)$ and dynamic range of geomagnetic pulsations derived from Memambetsu data $(\mathrm{MLT}=\mathrm{UT}+9)(b)$

Thus, this data allows us to conclude that the collision of a sequence of DSs forming the base of HPS with Earth's magnetosphere causes an increase in geomagnetic activity, and individual DSs trigger short-term increases in magnetic activity (substorms) coinciding with the duration of DS.

It is important to emphasize that $B_{\mathrm{z}}$ from 10:00 to 14:15 UT was northward $\left(B_{\text {zmean }}=3.27 \mathrm{nT}\right)$, but at 14:15 it made a short jump to the south (to $-4 \mathrm{nT}$ ) until 14:33 UT, and then again became steadily northward. The behavior of $B_{z}$ shows that in this case there was no long-term energy accumulation in the magnetotail due to reconnection, i.e. there was no substorm preliminary phase. The increase in auroral magnetic activity from 14:40 to 15:40 UT (Figure 3) can therefore be classified as sawtooth substorms [Troshichev et al., 2011; Troshichev, Janzhura, 2012].

\section{GEOMAGNETIC RESPONSE TO MAGNETOSHEATH DS RECORDED ON DECEMBER 23, 2002}

In this section, we will try to establish a relationship between the magnetosheath plasmoid [Karlsson et al., 2015] and SW DS and to estimate its effect on Earth's magnetosphere.

Figure 6 shows parameters of SW plasma and IMF from OMNI data for December 23, $2002(a)$ and for the magnetosheath plasmoid from Cluster $\mathrm{C} 1$ data [Karlsson et al., 2015] (b). Panel $a$ displays the $N$ and $B$ anticorrelation for $\sim 09: 56-09: 59$, which corresponds to SW DS. A plasmoid structure of similar type with a short time delay $(\sim 90$ s) appears in the magnetosheath.

In this case, $N$ and $B$ increase, and $V_{\mathrm{Sw}}$ decreases to $200 \mathrm{~km} / \mathrm{s}$. The time delay can be explained by the error in data transfer from the Wind orbit to Earth's orbit, as in the case of March 11, 2003. 
Table

SW diamagnetic plasmoids recorded in [Karlsson et al., 2015] and their features

\begin{tabular}{|c|c|c|c|c|c|c|c|}
\hline $\mathrm{N}$ & Date & UT & $V_{\mathrm{SW}}, \mathrm{km} / \mathrm{s}$ & SW type & Solar source & $A E, \mathrm{nT}$ & $B_{\mathrm{Z} \mathrm{GSM}}, \mathrm{nT}$ \\
\hline 1 & 2001.02 .12 & 16.2 & $400-430$ & slow SW & streamer belt & $52 / 129$ & 2.7 \\
\hline 2 & 2001.02 .12 & 16.3 & - & - & - & $50 / 100$ & 2.7 \\
\hline 3 & 2001.02 .12 & 18.3 & - & - & - & $50 / 120$ & 2,5 \\
\hline 4 & 2001.02 .12 & 21 & - & - & - & $270 / 430$ & -2.88 \\
\hline 5 & 2001.02 .12 & 21.7 & - & - & - & $342 / 555$ & -2.09 \\
\hline 6 & 2001.02 .12 & 23.5 & - & - & - & $84 / 127$ & 0.87 \\
\hline 7 & 2002.02 .02 & 1.1 & $340-410$ & slow SW & streamer chain & $110 / 145$ & -5.19 \\
\hline 8 & 2002.02 .02 & 15.5 & - & - & - & $100 / 155$ & -0.93 \\
\hline 9 & 2002.02 .02 & 2.5 & - & - & - & $96 / 268$ & 0.5 \\
\hline 10 & 2002.02 .02 & 7 & - & - & - & $504 / 660$ & -11.45 \\
\hline 11 & 2002.02 .02 & 13.8 & - & - & - & $291 / 347$ & -5.86 \\
\hline 12 & 2002.02 .02 & 14.1 & - & - & - & $320 / 359$ & -8.80 \\
\hline 13 & 2002.04 .12 & 22.7 & 410 & slow SW & streamer belt & $50 / 200$ & 2.55 \\
\hline 14 & 2003.01 .18 & 17 & 400 & slow SW & streamer belt & $135 / 270$ & 1.63 \\
\hline 15 & 2003.01 .29 & 12.7 & $410-440$ & slow SW & streamer belt & $702 / 814$ & -3.72 \\
\hline 16 & 2003.01 .29 & 13.4 & - & - & - & $436 / 800$ & -4.31 \\
\hline 17 & 2003.01 .29 & 18.9 & - & - & - & $543 / 691$ & -3.24 \\
\hline 18 & 2003.01 .29 & 19.6 & - & - & - & $440 / 607$ & -6.3 \\
\hline 19 & 2003.01 .29 & 19.8 & - & - & - & $441 / 548$ & -6.13 \\
\hline 20 & 2003.01 .29 & 22.2 & - & - & - & $614 / 825$ & -8.81 \\
\hline 21 & 2003.03 .11 & 14.6 & $400-420$ & slow SW & streamer belt & $170 / 310$ & -1.6 \\
\hline 22 & 2003.03 .11 & 17.3 & - & - & - & $80 / 180$ & 1.52 \\
\hline 23 & 2003.12 .31 & 11.5 & $420-230$ & slow SW & streamer belt & $500 / 600$ & -5.2 \\
\hline 24 & 2003.12 .31 & 19.9 & - & - & - & $200 / 439$ & 0.87 \\
\hline 25 & 2004.01 .02 & 15.9 & $430-440$ & slow SW & streamer chain & $480 / 100$ & 1.31 \\
\hline 26 & 2004.01 .21 & 22.9 & 450 & slow SW & streamer chain & $278 / 439$ & -2.33 \\
\hline 27 & 2004.04 .04 & 12.5 & $410-430$ & slow SW & streamer belt & $40 / 100$ & 6.15 \\
\hline 28 & 2004.04 .04 & 14.9 & - & - & - & $40 / 90$ & 10.73 \\
\hline 29 & 2004.04 .04 & 21.9 & - & - & - & $40 / 80$ & 9.44 \\
\hline 30 & 2005.02 .05 & 2.5 & 410 & slow SW & streamer belt & $20 / 50$ & 0.38 \\
\hline 31 & 2005.02 .14 & 7.6 & - & - & - & $60 / 120$ & -0.9 \\
\hline 32 & 2005.02 .16 & 12.3 & 420 & slow SW & streamer belt & $381 / 409$ & -2.9 \\
\hline 33 & 2005.02 .16 & 12.7 & - & - & - & $266 / 310$ & -2.9 \\
\hline 34 & 2005.02 .16 & 13.1 & - & - & - & $139 / 248$ & -2.8 \\
\hline 35 & 2005.02 .16 & 14 & - & - & - & $563 / 655$ & -2.85 \\
\hline 36 & 2005.02 .17 & 4.9 & 400 & slow SW & streamer belt & $96 / 191$ & 1.06 \\
\hline 37 & 2005.02 .21 & 13.3 & $370-420$ & slow SW & streamer belt & $200 / 280$ & -1.14 \\
\hline 38 & 2005.02 .21 & 14.5 & - & - & - & $200 / 250$ & -2.2 \\
\hline 39 & 2005.02 .21 & 20.8 & - & - & - & $63 / 105$ & -0.45 \\
\hline 40 & 2005.02 .21 & 20.9 & - & - & - & $60 / 110$ & -0.47 \\
\hline 41 & 2005.02 .21 & 21.1 & - & - & - & $35 / 125$ & -0.47 \\
\hline 42 & 2005.02 .21 & 21.4 & - & - & - & $39 / 58$ & -0.48 \\
\hline 43 & 2005.02 .21 & 21.5 & - & - & - & $37 / 58$ & -0.48 \\
\hline 44 & 2005.02 .22 & 1.3 & - & - & - & $15 / 50$ & 0.54 \\
\hline 45 & 2005.02 .22 & 3.8 & - & - & - & $50 / 90$ & 0.64 \\
\hline 46 & 2005.03 .05 & 10 & 390 & slow SW & streamer belt & $56 / 130$ & 1.35 \\
\hline 47 & 2005.03 .05 & 23.7 & - & & - & $535 / 481$ & 3.3 \\
\hline 48 & 2005.03 .13 & 5.4 & 350 & slow SW & streamer belt & $20 / 30$ & -1.1 \\
\hline 49 & 2006.02 .06 & 19 & 330 & slow SW & streamer belt & $200 / 250$ & -2.7 \\
\hline 50 & 2006.02 .06 & 19.5 & - & - & - & $200 / 300$ & -2.7 \\
\hline 51 & 2006.02 .19 & 2.5 & 370 & slow SW & streamer belt & $90 / 200$ & 0.99 \\
\hline 52 & 2006.02 .19 & 3.1 & - & - & - & $80 / 85$ & 0.67 \\
\hline 53 & 2006.02 .19 & 4.1 & - & - & - & $80 / 120$ & -0.32 \\
\hline 54 & 2006.02 .28 & 6.7 & 350 & slow SW & streamer belt & $12 / 31$ & 5.04 \\
\hline 55 & 2006.02 .28 & 6.9 & - & - & - & $12 / 31$ & 5.04 \\
\hline \multirow[t]{2}{*}{56} & 2006.04 .04 & 21.9 & 330 & slow SW & streamer belt & $380 / 597$ & -3.55 \\
\hline & & & & & & $A E_{\text {mean }}=202.5 / 295.6$ & $B_{\mathrm{z} \text { mean }}=-0.73$ \\
\hline
\end{tabular}




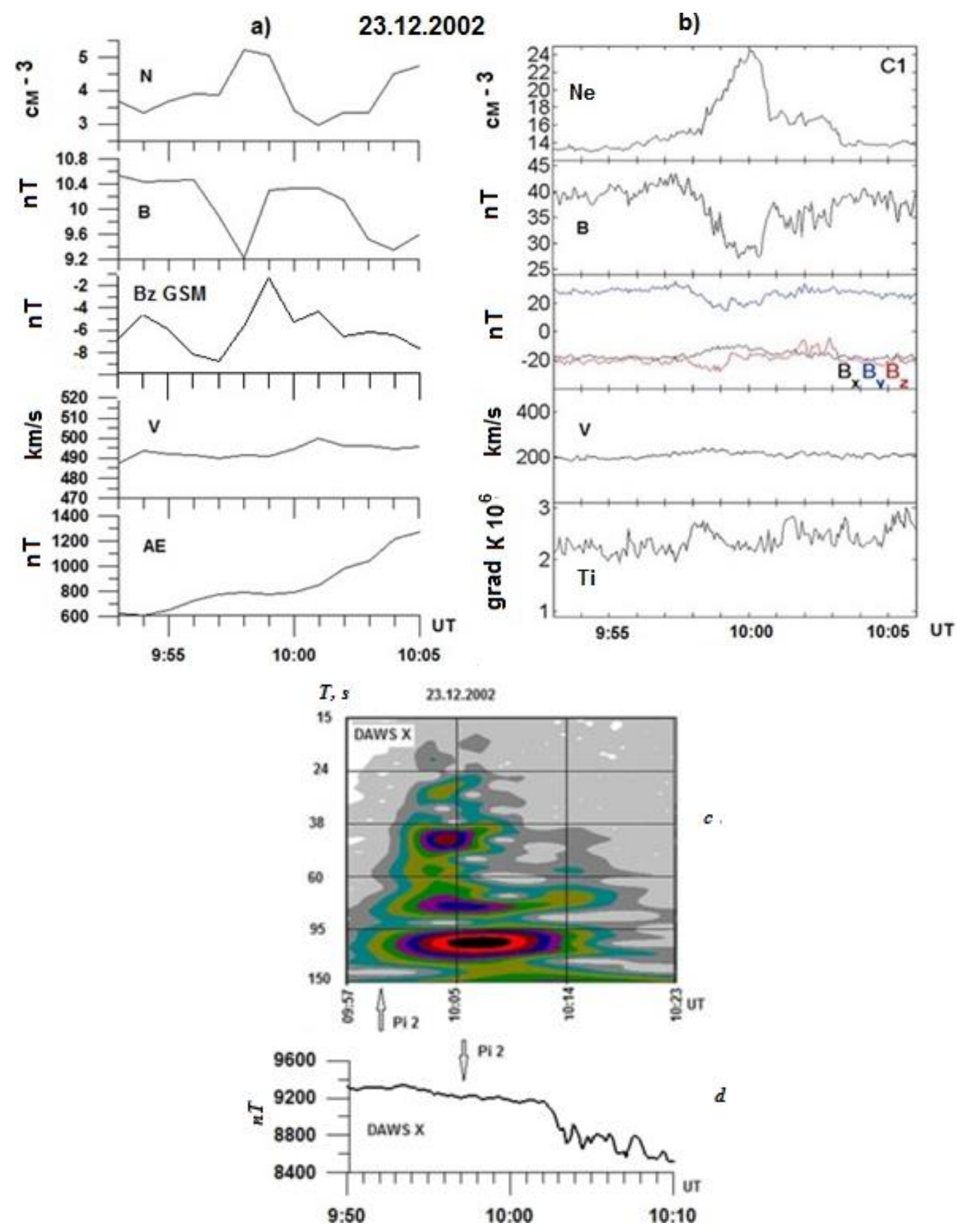

Figure 6. Variations in SW $N_{\mathrm{p}},|\mathbf{B}|, B_{\mathrm{z}}, V_{\mathrm{SW}}$, and $A E$ (OMNI data [http://cdaweb.gsfc.nasa.gov/cgi-bin/eval2.cgi]) (a); variations in $N_{\mathrm{e}},|\mathbf{B}|, B_{\mathrm{x}}, B_{\mathrm{y}}, B_{\mathrm{z}}, V$, and $T_{\mathrm{i}}$ in the magnetosheath on December 23, 2002 (data from [Karlson et al.,2015]) (b); dynamic spectrum of Pi2 geomagnetic pulsations from the Dawson Observatory (MLT = UT-10.3) (c) and fragment of a magnetogram from the same observatory $(d)$

All this means that the observed magnetosheath plasmoid is an extension of the DS detected in SW.

Terrestrial effects of the interaction of this SW DS in the transition layer are similar to the above effects of DS interaction. In the auroral zone in DAWS during midnight hours ( 23:58 LT), a burst of irregular geomagnetic pulsations $\mathrm{Pi} 2$ is recorded as an indicator of substorm onset, and at 10:02 UT the westward electrojet begins to sharply increase, reflecting in an $H$-component decrease by $\sim 800 \mathrm{nT}$ (Figure 6, $d$ ) and in an $A E$ increase to 1276 nT (Figure 6,a).

Unlike the previous case of March 11, 2003, the December 23, 2002 moderate magnetic disturbance oc- curred against high planetary and auroral magnetic activities $\left(K_{\mathrm{p}}=4+, A E\right.$ increased from 561 to $\left.1276 \mathrm{nT}\right)$. It is worth noting that on that day from 00 to 05 UT IMF $B_{z}$ was predominantly northward, the mean $B_{\mathrm{z}}$ and $A E$ were respectively 0.07 and $217 \mathrm{nT}$. At 05:00 UT, $B_{\mathrm{z}}$ became southward and remained so until 11:00 UT with $B_{\mathrm{z} \text { mean }}=-$ $4.78 \mathrm{nT}$. Within the interval from 09:50-10:10 UT when the plasmoid (DS) was recorded in the magnetosheath, the $A E$ index sharply increased to $1276 \mathrm{nT}\left(A E_{\text {mean }}=885\right.$ $\mathrm{nT}$ ) with the same $B_{\mathrm{z} \text { mean }}=-5.0$ in this interval.

Thus, we can conclude that DSs and plasmoids are identical and geoeffective. In Earth' orbit, a source of DS in the March 11, 2003 event is the streamer belt or HPS. 
Diamagnetic structures associated with the streamer chain of August 01, 1998 and the response of Earth's magnetosphere to their impact. Consider another example of the global geomagnetic response of the magnetosphere to the interaction with DS whose source is the streamer chain.

Figure 7, $a$ shows the results of calculations, made by Rudenko [http://bdm.iszf.irk.ru/] in the potential approximation, of positions of footpoints of open magnetic tubes corresponding to coronal holes in spherical coordinates for July 24, 1998 ( 14:35 UT). Panel $b$ for the same date and the same time presents the CR1938 synoptic map of the calculated NL of the radial component of the global magnetic field on the source surface $\left(R=2.5 R_{\odot}\right)$ (solid curve); "+" is the positive field polarity from the Sun; "-“", to the Sun. The dashed line indicates calculated streamer chains separating bases of coronal holes with the same direction of the solar magnetic field. We are interested in the area of intersection between streamer chain and ecliptic on the Sun denoted on the synoptic map presented in panel $b$ by letter $\mathrm{O}$ (near a longitude of $50^{\circ}$ ) with a vertical arrow. This area separates the bases of coronal holes, designated by $\mathrm{d}$ and $\mathrm{e}$ in panel $a$. Position of the central meridian at the time considered is shown by the vertical dotted line (panel $b$ ).

Due to the solar rotation, as seen in panel $a$, the point $\mathrm{O}$ intersects the central meridian when the latter moves to the left in longitude by $\sim 50^{\circ}$, which corresponds to the time interval of $\sim 3$ days $18 \mathrm{hrs}$ (the Sun rotates by $13.3^{\circ}$ per 1 day). This corresponds to $t_{0}=09: 00$ UT (July 28, 1998) when the point O is on the central meridian. In Earth's orbit, the streamer chain area closest in time with an even number of changes of the IMF azimuth angle sign (Figure 8, a), a higher density $N \sim 20-30 \mathrm{~cm}^{-3}$ (Figure $8, b$ ), and a relatively low velocity $V_{\mathrm{SW}} \sim 460 \mathrm{~km} / \mathrm{s}$ (Figure $8, c$ ) was observed at $1 A U$ on August 01, 1998 between $\sim 18: 05$ and 18:35 UT. Using the formula $t_{\text {Earth }} \approx t_{0}+4.6 \cdot 10^{4} / V$, we obtain that at $V \sim 460 \mathrm{~km} / \mathrm{s}$ the area "O" reaches Earth on August 01, 1998 at 13:00 UT. $\Delta t_{\text {Earth }} \approx 4$ days $4 \mathrm{hrs}$. Hence, $t_{\text {Earth }}=13: 00$ UT (August 01, 1998). This accurate to $\sim 5 \mathrm{hrs}$ (a relative accuracy of $\sim 5 \%$ ) is consistent with the observed time of occurrence of the streamer chain area at $1 \mathrm{AU}$ (Figure 8).
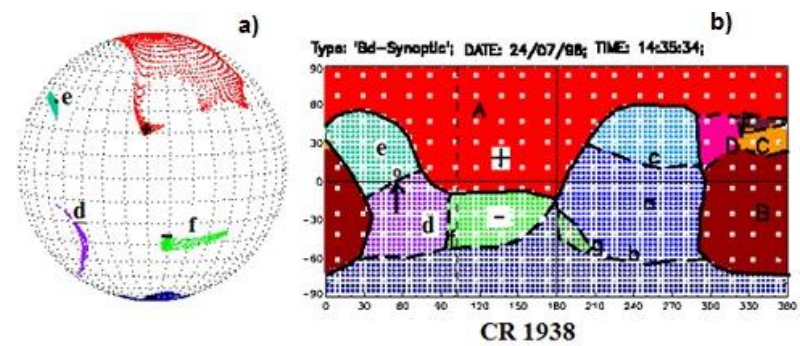

Figure 7. Results of calculations made by Rudenko [http://bdm.iszf. irk.ru/] in the potential approximation: Positions of footpoints of open magnetic tubes corresponding to coronal holes in spherical coordinates for July 24, 1998 ( 14:35 UT) (a); CR1938 synoptic map of the position of neutral line (solid curve) and streamer chains (dashed line) on the source surface $(b)$

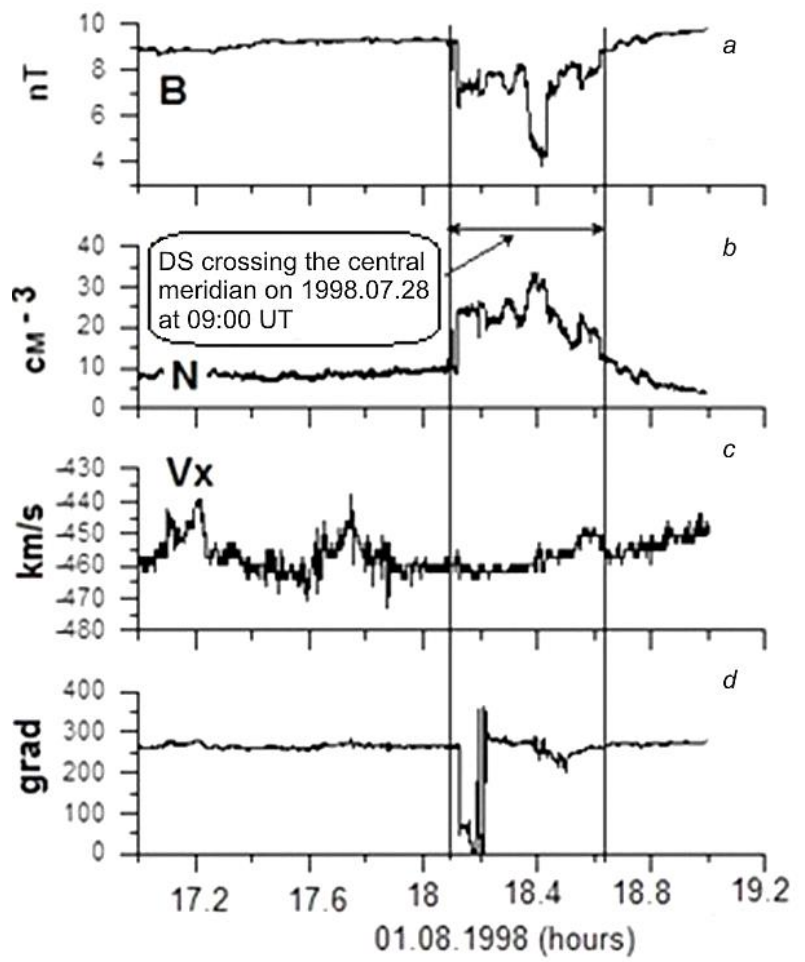

Figure 8. Parameters of IMF and SW plasma for August 01, 1998: $|\mathbf{B}|$ is the IMF modulus $(a), N$ is the SW plasma density $(b), V_{\mathrm{x}}$ is the SW velocity $(c), \Phi$ is the IMF azimuth angle $(d)$. The streamer belt area in Earth's orbit is shown by vertical lines. OMNI data [http://cdaweb. gsfc.nasa.gov/cgibin/eval2.cgi]

From the $B$ and $N$ anticorrelation in Figure $8, a, b$ $(R=-0.95)$ it follows that the streamer chain area in Earth's orbit is DS within which embedded DSs with decreasing transverse size are recorded (as is the case with the previously discussed streamer belt area (HPS) at $1 \mathrm{AU})$.

The interaction between these DSs and the magnetosphere leads to an increase in magnetic activity reflecting in a sharp increase followed by a wave-like $A E$ variation (Figure 9, a). Such $A E$ variations are peculiar to sawtooth substorms. Such substorms have no welldefined growth phase [Troshichev et al., 2011, Troshichev, Janzhura, 2012]. The $A E$ modulation in such substorms is associated with synchronous antiphase variations in $N$ and $|\mathbf{B}|$ in DS [Parkhomov et al., 2018].

The first attempt to analyze the magnetospheric response to interaction with plasma pressure jumps associated with DSs forming the base of the streamer chain area of August 01, 1998 was made in [Parkhomov et al., 2011]). Results of this analysis allowed the authors to suggest that in this event due to this interaction there is a mechanism of direct energy transfer from SW to the auroral zone simultaneously through two channels: through the induced electric field and through the compressional amplification of the magnetospheric current system.

To date, according to the conclusions drawn in [Parkhomov et al., 2017; Parkhomov et al., 2018] as well as to the results of the brief analysis carried out at the beginning of this Section, the following becomes clear: the plasma pressure jumps recorded in the August 01, 1998 event 

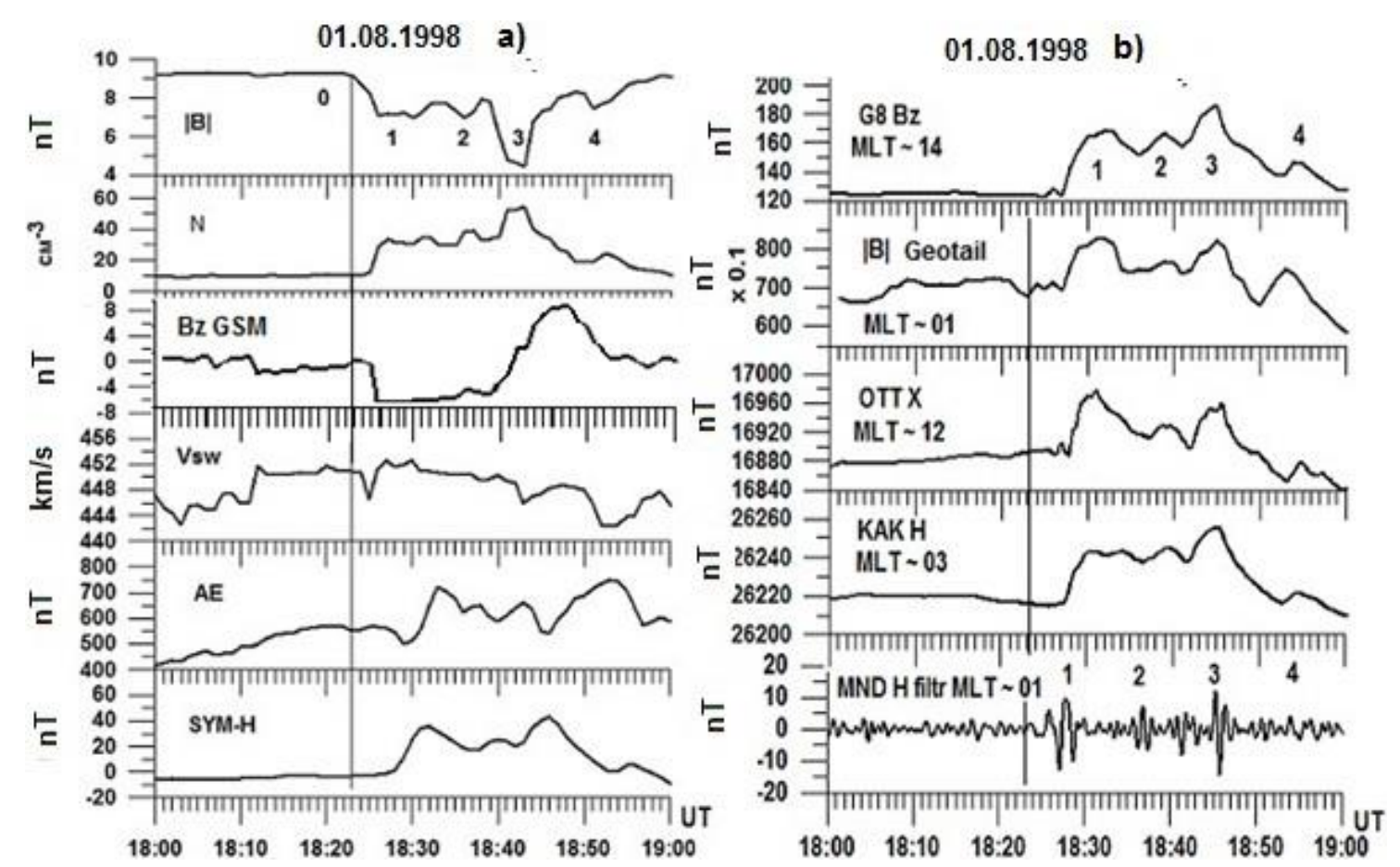

Figure 9. From top to bottom: variations in $|\mathbf{B}|, N, B_{7}$, and $V_{\mathrm{SW}}$ in DS related to the streamer chain, $A E$ and SYM-H from OMNI data [http://cdaweb.gsfc.nasa.gov/cgi-bin/eval2.cgi] (a); complex of geophysical phenomena reflecting the magnetospheric response to interaction with DS $(b)$. From top to bottom: $B_{\mathrm{z}}$ of the geomagnetic field in the GOES-8 geostationary orbit; $|\mathbf{B}|$ of the geomagnetic field in the magnetotail from Geotail data $\left(\sim 10 R_{\mathrm{E}}\right)$; the horizontal component $H$ of the geomagnetic field from the Ottawa Observatory (OTT) data for midday hours LT; from the Kakioka Observatory (KAK) data for post-midnight hours LT; $H$-component oscillogram from the induction magnetometer of the Mondy Observatory (MND) at near-midnight hours (the initial recording is digitally filtered by a narrowband filter). Numbers correspond to the numbers of smaller-scale embedded DSs

[Parkhomov et al., 2011] correspond to the plasma density jumps at boundaries of DSs constituting the base of the streamer chain area in Earth's orbit.

Turning to the analysis of the interaction of the DS sequence with the magnetosphere, let us look at Figure 9. The first general analysis shows that the collision of the DS sequence associated with the streamer chain on August 01, 1998 within the interval $\approx 18: 23-19$ :00 UT caused a global increase in geomagnetic and auroral activities. This increase manifested itself in a synchronous occurrence of various geophysical phenomena on the day and night sides of Earth, in the geostationary orbit, and in the central part of the plasma sheet of the magnetotail during midnight hours. Looking more closely at panel $a$, we can see increases in $K_{\mathrm{p}}$ from $4+$ to $6-$; $A E$, from 500 to $800 \mathrm{nT}$; SYM-H, from -10 to $+40 \mathrm{nT}$.

The variations in the indices are synchronous with the variations in the magnetic field and SW density. The correlation coefficients $R_{A E, N}=0.48, R_{S Y M-H, N}=0.79$, but the correlation is worse with the $B$ modulus $R_{B, A E}=-0.44$, $R_{B, S Y M-H}=-0.68$.

The PilB geomagnetic pulsations peculiar to the substorm explosive phase in the near-midnight sector of the auroral zone occurred at low latitudes synchronously with phenomena on the day side (Figure 10). With the period of $N, B$ variations in SW there was also a global modulation of variations in ionospheric currents and global induced magnetic field obtained from calculations of current vectors at the worldwide network of magnetic observatories [Parkhomov et al., 2011].
Abrupt jumps of SW density and IMF components, associated with DS, generated a substorm, which consisted of four activations of aurora on the night side (denoted by numbers 1-4 that mark the embedded DSs in Figure 9) (Figure 11). It is important to note here that the activations of aurora on the night side were preceded by short-term bursts of aurora on the day side (number $0)$. Substorm activations of aurora in the eveningmidnight sector were caused by the interaction with small-scale DSs embedded into the larger-scale DS associated with the streamer chain.

\section{RESULTS}

The above studies have shown that the SW diamagnetic plasmoids observed in the slow SW examined in [Karlsson et al., 2012, 2015] are internal elements of fractal DSs of the slow SW. They represent a sequence of smaller-scale embedded DSs. The analysis of the dynamics of ground phenomena accompanying the interaction between the sequence of embedded DSs and the magnetosphere allows us to assign them to sawtooth substorms [Troshichev et al., 2011, Troshichev, Janzhura, 2012]. We should also recall here that DS boundaries in Earth's orbit feature a dynamic pressure jump $P_{\mathrm{d}}$ whose effects on the magnetosphere have been discussed in detail in [Keika et al., 2009] for the June 21, 2009 event and for a much larger number of events in [Zhou, Tsurutani, 2001]. Conclusions drawn in these works confirm the results of our analysis, which has also 


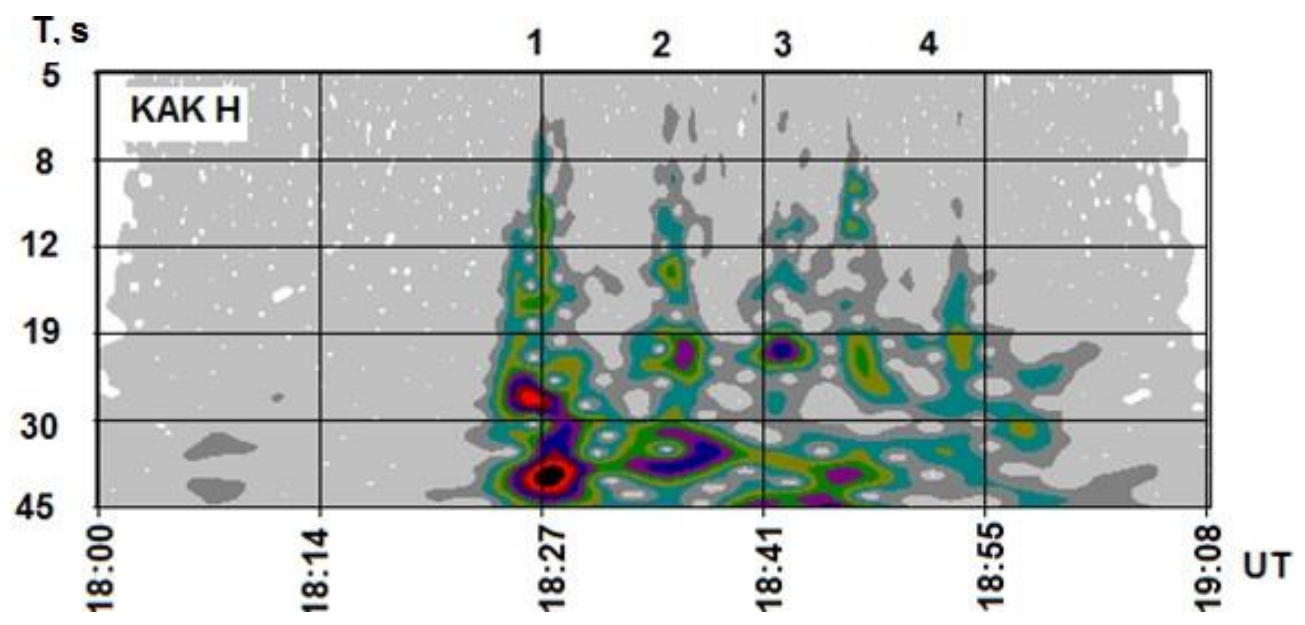

Figure 10. Dynamic spectrum of geomagnetic pulsations derived from KAK data for post-midnight hours (MLT= UT +9$)$. Numbers correspond to Figure 9

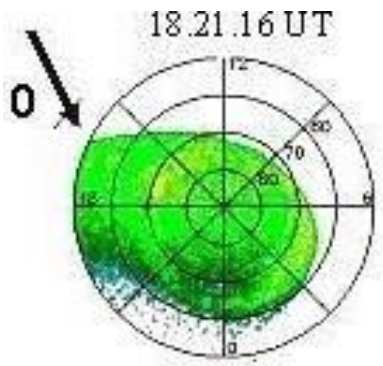

18.27.24 UT

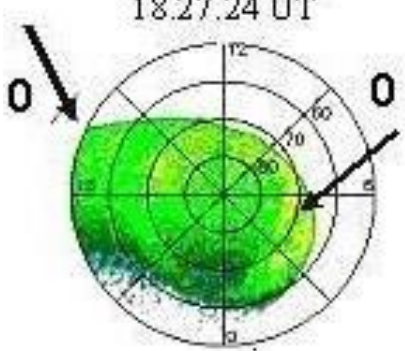

18.30.28 UT

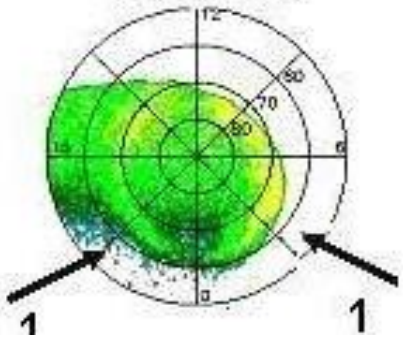

18.33.32 UT

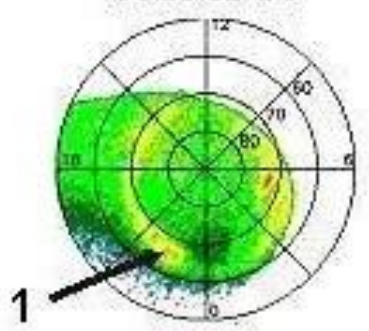

18.34.36 UT

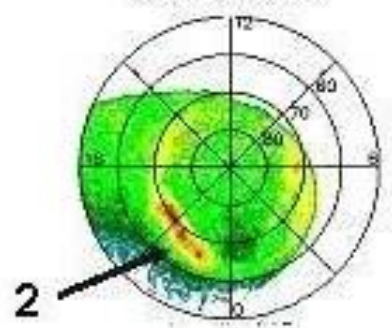

18.39.40 UT

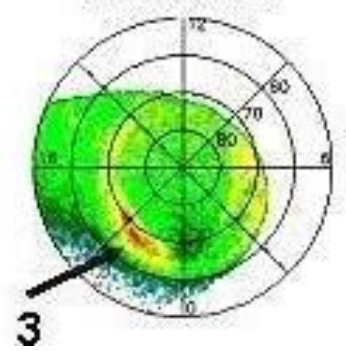

18.47 .39 UT

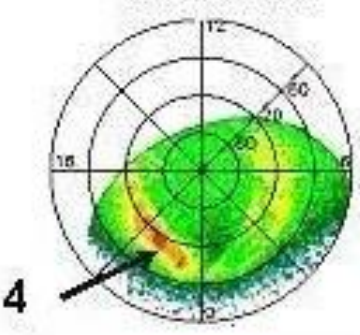

18.50.43 UT
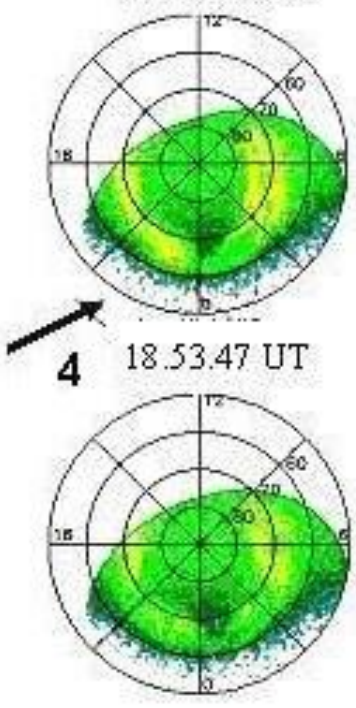

18.55.00UT

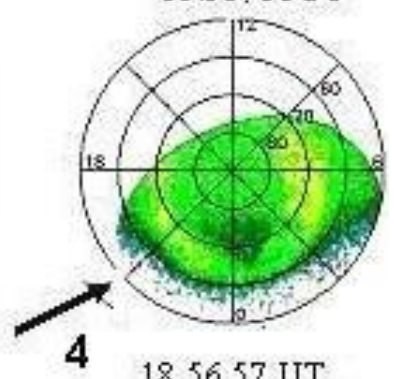

\section{4}

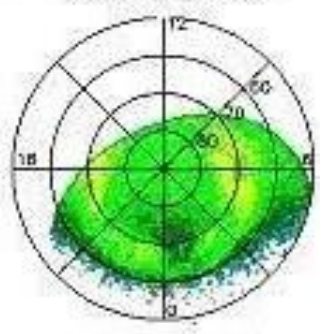

$18.59 .55 \mathrm{UT}$

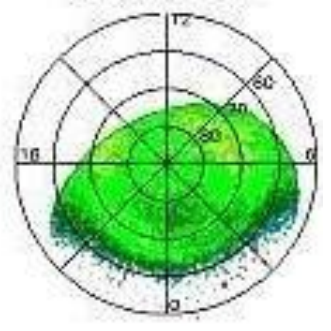

Figure 11. Auroral oval images taken by an UVI camera with LBHL filter on board the Polar satellite on August 01, 1998, which demonstrate the magnetospheric response to the DS sequence in the form of a sawtooth substorm. Numbers denote activations of aurora corresponding to DSs numerated in Figure 9

also shown that DSs can similarly affect the magnetosphere at both positive and slightly negative values of preceding IMF $B_{\mathrm{z}}$. The presence of negative $B_{\mathrm{z}}$ leads to an increase in $A E$ and $S Y M-H$ jumps as compared to the previous positive $B_{\mathrm{z}}$.

The short-term activations of geomagnetic and auroral activity coincide with DS in duration, i.e. the sequence of sawtooth substorms was generated by phenomena in SW rather than in the magnetotail (Fig- ures 9-11) or by a direct impact of SW DS through the shock front and magnetosheath on the magnetosphere due to the direct energy transfer from SW to the magnetosphere and auroral zone.

Let us discuss the occurrence of the geomagnetic response to the interaction between DS of the slow SW and Earth's magnetosphere, using the results obtained in [Kozyra et al., 2013]. The authors have examined the January 21, 2005 superstorm triggered by the interaction 
of Earth's magnetosphere with DS, which was associated with the filament in the sporadic SW rather than with the slow SW.

The authors have shown that the material of the dense solar filament was captured into the magnetosphere at the northward IMF component. In the place, where this absorption occurred, the plasma density of the magnetosphere increased to $130 \mathrm{~cm}^{-3}$, the temperature increased by $1-2 \mathrm{keV}$, and IMF turned to the north. Due to the double high-latitude reconnection, the area of magnetosheath tubes filled by solar filament plasma was transformed into a single layer of magnetospheric tubes with fluxes of the captured filament material. As a result, a cold dense plasma sheet (CDPS) was formed in the magnetosphere.

Simulation and observations made by the authors have shown that for $1 \mathrm{hr}$ the plasma sheet density at midnight reached $6 \mathrm{~cm}^{-3}$; in the geostationary orbit, tens of $\mathrm{cm}^{-3}$; at sunset and sunrise, from tens to hundreds of $\mathrm{cm}^{-3}$ in the low-latitude boundary plasma layer (LLBL)

In the cases we analyzed, jumps of density and magnetic field modulus were comparable with jumps in the event analyzed in [Kozyra et al., 2013]. The DSmagnetosphere interaction also occurred at predominantly northward IMF vertical component. All this attests to the fact that the mechanism of DSmagnetosphere interaction in our case could be similar to that of the January 21, 2005 event [Kozyra et al., 2013].

\section{CONCLUSIONS}

1. Diamagnetic structures of the slow SW in Earth's orbit are recorded as a sequence of DSs with different scales.

2. SW diamagnetic plasmoids, defined in [Karlsson et al., 2012, 2015], are small-scale DSs of the slow SW.

3. Collision of the slow-SW DS sequence with Earth's magnetosphere causes geomagnetic activity to increase, and individual DSs lead to short-term increases in magnetic activity (substorms) approximately coinciding with the duration of DS impact.

4. The mechanism of the interaction of slow-SW DSs at the northward IMF component can be associated with the capture of higher density plasma constituting DS to the magnetosphere.

We are indebted to G.V. Rudenko for the calculation of positions of footpoints of open magnetic tubes of coronal hole and neutral line of the global solar magnetic field. We are grateful to the referee for constructive comments, possibility of contacting with Dr. T. Karlsson, and for access to the data. We greatly appreciate NASA CDAWEB providing ACE, IMP-8, Wind, Geotail, Polar, GOES-8, GOES-10 data. We thank managers, developers of the instruments and leaders of experiments, conducted with these satellites, for the possibility of using the data. Thanks are extended to I.R. Mann, D.K. Milling, and other members of the CARISMA team for providing data.

The work was performed as part of State task No. 00700163-18-00 of January 12, 2018 and joint RussianTaiwan RFBR project MOST-107-2923-M-008-001MY3/18-55-52006 MHTa.
Participation of Parkhomov V.A. in this work was supported by RFBR grant as part of Research Project No.18-55-52006 MHTa; and of Dmitriev A.V., by grant MOST 107-2111-M-008-011.

\section{REFERENCES}

Borrini G., Wilcox J.M., Gosling J.T., Bame S.J., Feldman W.C. Solar wind helium and hydrogen structure near the heliospheric current sheet; a signal of coronal streamer at 1 AU. $J$. Geophys. Res. 1981, vol. 86, pp. 4565.

Bostick W.H. Experimental study of ionized matter projected across a magnetic field. Phys. Rev. 1956, vol. 104, pp. 292-299.

Eselevich M.V., Eselevich V.G. Fractal structure of the heliospheric plasma sheet in the Earth's orbit. Geomagnetism and Aeronomy. 2005, vol. 45, no. 3, pp. 326-336.

Eselevich, M.V., Eselevich V.G. Manifestations of the ray structure of the coronal streamer belt in the form of sharp peaks of the solar wind plasma density in the Earth's orbit. Geomagnetism and Aeronomy. 2006, vol. 46, iss. 6, pp. 770-782.

Eselevich V.G., Fainshtein V.G. The heliospheric current sheet (HCS) and high-speed solar wind: interaction effects. Planetary Space Sci. 1991. V. 39. P. 737-744.

Eselevich V.G., Fainshtein V.G. On the existence of the heliospheric current sheet without a neutral line, Planet. Space Sci. 1992. V. 40. P. 105.

Eselevich M.V., Eselevich V.G. The double structure of the coronal streamer belt. Solar Phys. 2006, vol. 235, iss. 1-2, pp. 331-344.

Eselevich V.G., Fainshtein V.G., Rudenko G.V. Study of the structure of streamer belts and chains in the solar corona. Solar Phys. 1999, vol. 188, pp. 277.

Eselevich M., Eselevich V., Fujiki K. Streamer belt and chains as the main sources of quasi-stationary slow solar wind. Solar Phys. 2007, vol. 240, pp. 135-151. DOI: 10.1007/s11207-006-0197-z.

Ivanov K., Bothmer V., Cargill P.J., Kharshiladze A., Romashets E.P., Veselovsky I.S. Subsector structure of the interplanetary space. Proc. The Second Solar Cycle and Space Whether Euroconference. Vicvo Equense, Italy. 2002, p. 317.

Karlsson T., Brenning N., Nilsson H., Trotignon J.-G., Vallieres X., Facsko G. Localized density enhancements in the magnetosheath: Three-dimensional morphology and possible importance for impulsive penetration. J. Geophys. Res . 2012, vol. 117, no. A03227. DOI: 10.1029/2011JA017059.

Karlsson T., Kullen A., Liljeblad E., Brenning N., Nilsson H., Gunell H., Hamrin M. On the origin of magnetosheath plasmoids and their relation to magnetosheath jets J. Geophys. Res.: Space Phys. 2015, vol. 120, iss. 9, pp. 7390-7403. DOI: 10.1002/2015JA021487

Keika K., Nakamura R., Baumjohann W., Angelopoulos V., Chi P.J., Glassmeier K.H., et al. Substorm expansion triggered by a sudden impulse front propagating from the dayside magnetopause. J. Geophys. Res. 2009, vol. 114, no. A00C24. DOI: 10.1029/2008JA013445.

King J.H., Papitashvili N. One min and 5-min solar wind data sets at the Earth's bow shock nose // One min and 5-min solar wind data sets at the Earth's bow shock nose. URL: https://omniweb. gsfc.nasa.gov/html/HROdocum.html. Joe King and Natalia Papitashvili, GSFC/SPDF and ADNET Systems, Inc. (accessed January 16, 2019).

Korzhov N.P. Large-scale three-dimensional structure of the interplanetary magnetic field. Solar Phys. 1977, vol. 55, pp. 505.

Kozyra J.U., Liemohn M.W., Cattell C., Zeeuw D.De, Escoubet C.P., Evans D.S., et al. Solar filament impact on 21 January 2005: Geospace consequences. J. Geophys. Res.: Space Phys. 2013, vol. 119, pp. 5401-5448. DOI: 10.1002/2013JA019748. 
Lyons L.R., Lee D.-Y., Wang C.-P., Mende S.B. Global auroral responses to abrupt solar wind changes: Dynamic pressure, substorm, and null events. J. Geophys. Res. 2005 , vol. 110, no. A08208. DOI: 10.1029/2005JA011089.

Parkhomov V.A., Borodkova N.L., Dmitriev A.V., Klimov P.M., Rakhmatulin R.A. The role of solar wind pressure jumps in the initiation and control processes of magnetospheric substorms. Geomagnetism and Aeronomy, 2011, vol. 51, no. 7, pp. 979-993.

Parkhomov V.A., Borodkova N.L., Eselevich V.G., Eselevich M.V. Abrupt changes of density in sporadic solar wind and their effect on Earth magnetosphere. Cosmic Res. 2015, vol. 53, no. 6, pp. 411-422. DOI: 10.1134/S0010952515050093.

Parkhomov V.A., Borodkova N.L., Eselevich V.G., Eselevich M.V. Features of the impact of the solar wind diamagnetic structure on Earth's magnetosphere. Solar-Terrestrial Physics. 2017.vol. 3, iss. 4 pp. 44-57. DOI 10.12737/szf-34201705.

Parkhomov V.A., Borodkova N.L., Eselevich V.G., Eselevich M.V., Dmitriev A.V., Chilikin V.E. Solar wind diamagnetic structures as a source of substorm-like disturbances. J. Atm. and Solar-Terr. Phys. 2018, vol. 181, pp. 55-67. DOI: $10.1016 /$ j.jastp.2018.10.010.

Svalgaard L.J., Wilcox W., Duvall T.L. A model combining the solar magnetic field. Solar Phys. 1974, vol. 37, pp. 157.

Troshichev O.A., Kan Liou, Stauning Peter, Reeves G. Saw-tooth substorms: inconsistency of repetitive bay-like magnetic disturbances with behavior of aurora. Adv. Space
Res. 2011, vol. 47, no. 4, pp. 702-709. DOI: 10.1016/j.asr. 2010.09.026.

Troshichev O.A, Janzhura A. Space Weather Monitoring by Ground-Based Means: PC Index. Springer Verlag, 2012. 287 p. DOI: 10.1007/978-3-642-16803-1.

Wang Y.M., Sheeley N.R., Rich N.B. Coronal pseudostreamers. The Astrophys. J. 2007, vol. 685, pp. 1340.

Winterhalter D., Smith E.J., Burton M. E., Murphy N. The heliospheric plasma sheet J. Geophys. Res. 1994, vol. 99, pp. 6667.

Zhou X., Tsurutani B.T. Interplanetary shock triggering of nightside geomagnetic activity: Substorms, pseudobreakups, and quiescent events. J. Geophys. Res. 2001, vol. 106, pp. 18,957 .

URL: http://wso.stan-ford.edu/ (accessed August 12, 2019).

URL: http://bdm.iszf.irk.ru (accessed August 10, 2019).

URL: http://cdaweb.gsfc.nasa.gov/cgi-bin/eval2.cgi (accessed August 12, 2019).

\section{How to cite this article}

Parkhomov V.A., Eselevich V.G., Eselevich M.V., Dmitriev A.V., Vedernikova T.I. Diamagnetic plasmoids as part of diamagnetic structures of the slow solar wind and their impact on Earth's magnetosphere. SolarTerrestrial Physics. 2019. Vol. 5. Iss. 4. P. 34-45. DOI: 10.12737/stp54201905. 